\title{
Inḩalts= Derzeidnis
}

I. Bräjen ritite, Borperjammlung Tagejoronumg uiw.

II. Berbandlungen bes Bsenoffenidaft stages.

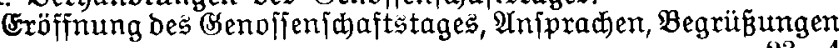

Beridft bes $\mathfrak{U}$ nwalts .

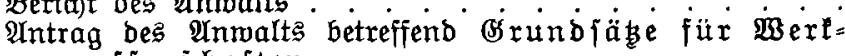
genofjenifiaften ............ . . 63-86

Dte Sanbwerfergenoffenfajaften in Baben. . . . . . . 86-107

Uustaufd) von (Erfabrumgen tm Ssejdäftabetrieb (ber ફ̧and= merfergendfenffjaften) . . . . . . . . . . 107-109

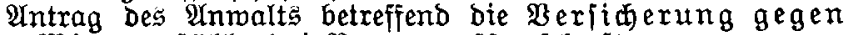
Mietauffäle bei $\mathfrak{B a u g e n o f f e n f d i f t e n . 1 1 0 - 1 2 9}$

Untrag Des Yrmalts betreffend bie Bujammenjebung der Bereinsorgane und die $\mathfrak{B a b l}$ ber $\mathfrak{B a r}$ tand jidtsratsmitglieber in $\mathfrak{B a u g e n o f f e n f d a f t e n ~ - ~} 3 \mathfrak{a h l}$ ber Borftanosmitglieber

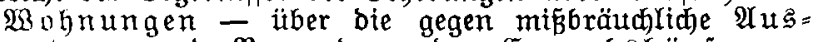
nubung unb Bermenoung ber Ermerbshäufer ge= troffenen Makregeln

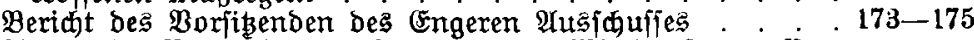

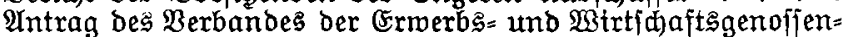
(đ)aften am Mittelrbein betreffend bie Anlegung von Gandbibliothefen feitens der Genojenfáften. 176-181

Intrag des Berbandes Der Unterbabifden Freditgenoffen= fifaften betreffend bie nidht exforberltae gejebliche Regelung bes Depoften= und Spartafienmefens 181-200

Untrag bes unmalts betreffend die (s) $x$ und fäbe für bilanz=

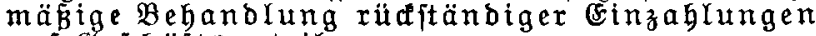
auf (o)

Wablen in Den Borftand Der Heilfataffe nad \& 8 bes Statuts ber Silfalaffe . . . . . . . . . . . . . . . .

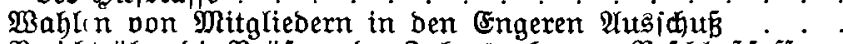

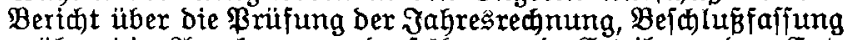
über die Anertennung berfelben und Erteilung ber Ent= laftung

Beriđt itber bie Einnahmen und

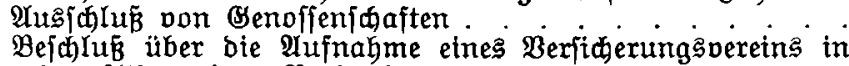
ben Ullgemeinen Berband . .

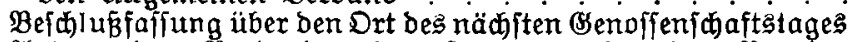

Antraa Des $\mathfrak{B e r b a n b e s}$ der Ronlumbereine Der Brovinz Sadjen und ber angrenzenben Provinzen und Staaten betreffend $\mathscr{R}$ iquibität $\delta$ er $\Re$ onfumvereine. . . . 214-236

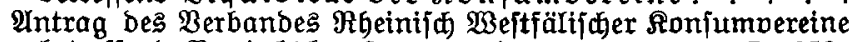
betreffend $B$ ezitistonjumvereine..2. 2:37-252

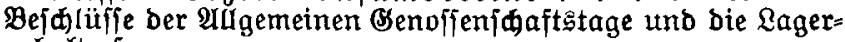
balterfrage.................. 254-261 
Intrag bes Inwalts betreffenb bie Dtslontierung von

Bufforberungen . . . . . . . . . 262-291 (338)

Antrag Des Berbanbes ber Rrebitweretne ou Berltn gegen bie

Brinbung von $5 a u s b e f t$ er. Srebitgenoffen waften 291-30s

Intrag bes Qnnwalts betreffenb bie Etnfllgeung etner $\mathfrak{B} e=$

Iaftung I Ifte bet ben frebitgenoffenjoaften . . . . . 303-310

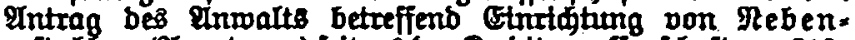

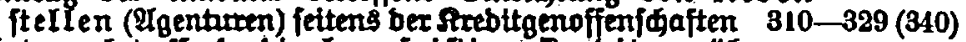

Intrag betreffend ble Iangfrtftge Rrebitgewäbrung

[zurit d gefteIIt)

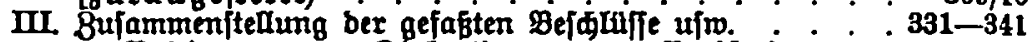

- Bebingungen: Dlstontterung von Bufforberingen

- Tebenftellen (Iggentaren) . . . . . . . 838/340

IV. Bertat über bie Berbanblungen ber Derbanbsareviforen :342-361

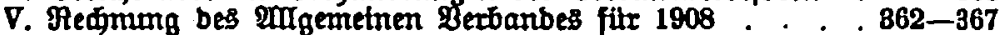
Boranjhläge für 1909 und 1910 . . . . . . 368-370

VI. Beridt liber ititglieberbeftand und intitieberberogung bes gagemetnen Verbambes $. . .370-373$

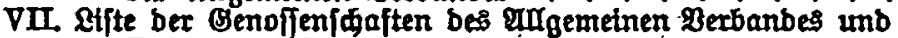
Bettrags It fte . . . . . . . . . . . . . 374-438

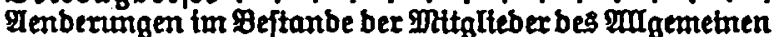
Berbanbes wäbrenb bes Jabres 1409 . . . . . 438/439 Feltbetträge ufw., 8ufammenftellung ber Be famtbetträge.

VIII. Zujammenftellumg ber uniterberbänbe 


\section{Derzeichnis der Redner.}

Selte

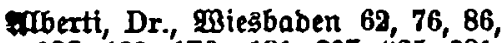
$128,139,176-181,207$, 235, 281 , 324,355

Inbuhl, Infterburg . . . . 325

\$ađ, Mtinden . . . . . . 235 Beder, Dr., EGarlottenburg 204 btg $207,209,237-247$

Bertfolb, Blumentbá 109, 124, 127, $129,138,139,145,148,151,152$, $161,165,167,168,169,171,172$, $350,351,352$

Creceltus, Çaxlottenburg 152-161, $168,171,262-276,310-320,345$ bis 349, 352, 353

Erüger, Dr., (ธ̧arLottenburg, 42-62, $76-81,83,48-103,104,107,14:$ $148,151,195-200,212,214,229$ bis 233, 234, 235, 247-249, 251, $252,254-261,28 i-285,286,291$, $302,308,309,326,328,329,942$, $344,345,352,354,359,360$

Dörr, Friebberg i. Đefien 356, 360

Zeierabenb, Mündien . 126, 148

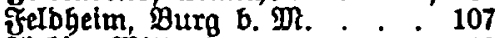
Ftebtg, פittenberge . : 148 Ұ゙la do, Muinden . $357,35 \dot{3}, 360$

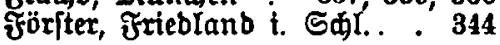

Grabler, Böpnect . . . . . 22 Grumer, Pforzheim . . . . 235

Eanjen, Sitel 127, 139, 163, 170, 172

Jenโen, fieL=Baarben . . . 150

Qeibel, Stargarb . . . . 360

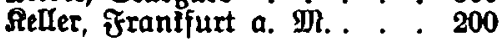

Seite

SIlette, BreșLau . . • . . 233 尺urr, Stettin 16, 191, 330, 35i, 352, $355,357,359$

giebelt, \&ifja i. \$. . . . . . 329 Robr, RarIsarube t. $\mathfrak{B} . \quad 87-98,105$ Sorenzen, St. Ingbert . . 357

SRager, Berltn . 81, 103, 289, 301 Malz, frantfurt a. $\mathfrak{T}$. 276-279, 285, 851, 855, 856 Marggraff, Mirdorf . . . 357 Mlaudex, Sarlşrube 227-229, 234 Meijnex, Dr., BerIin 201-204, 209 Mithelmann, BIantenburga. 5 arz 360 Dhâler, Met. . . . . 146-148

Reugебаиег, Breฐโаu 126, 233, 276, 302, 355, 360 NoIte, Rübenjøeib . 249-251, 252

Oppermann, Magbeburg 211, 212, $213,214,237,247,252,261$

Beter, Rarlśsube t. $\mathfrak{B} .181-190,200$ Bfiterer, Freiburg i. $\mathfrak{B}$. $\quad .29$ \$roeb|t, פuinden 23-26, 29, 32, $39,4 \mathrm{l}, 60-62,173-176,181,190$, $192,200,209,210,211,212,213,214$

Aabetyge, Berlin 165-167, 168, 172, Meban, Ellerbet. . . . . . $\quad \begin{aligned} & 351,352 \\ & 167\end{aligned}$ Nente, জannover . . . . . 280 Pldter, Dr., BerItn .. .. . . 27 Plidter, wien . . . . . . 39 Nöttinger, Fretburg t. . . . . 16

Pues, Eaffel . . . . . . 86

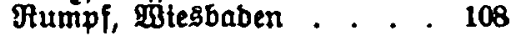


Selte

Eaenger, Dtersgeim b. RegI. 40

Sڤetbt, ఫannovex, $110-124,127$,

Stoll, Bab Slaubetu . . . 213

Stilben, Perlin yy1-301, 302, 360

Zart[ø, Extona a. E. . . . 279

SGIapplohL, ELlerbel 127, 144, 145

Śmibt, đrantfurt a. gir. 126, 150

Sqnetber, Dr., Potsbam, 145, 151, $215-522,236,344,355,360$

ङळäbon, BItetmis . . . 356

Sकuly, Eafाel $143,16 t, 168,350,353$

Sdroeber, Dr., EafTel 124, 128, 134, $140,150,151,161-163,164,352$,

359,361

Sळumaळer, Stuttgart 63-76,88 Getbert, \$3tesbaben 303-308, 344, $351,356,359$

Soexten Bexlin. . . . 356 Spib, Eojel . 194, 320-321 Stabler, Jeftetten . . . . 16

Stoff, Petnidemborf . . . . 190

Hibel, Jreiburg i. $\mathscr{O}$. . . . . . 33

3oglex, Dr., $33 i e n$ 38, 191, 287-289

Bolgt, ELberfelb . . . 141, 170

seinmann, 2ugg6urg . 327, 398

Prela, Breslau 2:22-227, 254, 236

girfer, farlorube . . . . 360

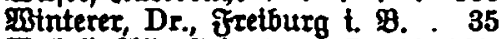

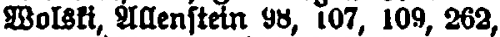
276, 279, 290, 291, 303, 309, 310 , $829,330,342,3+5,357,361$

פrabes, פ̇len 37, 84, 192-194, 207

bis 209

3abemaథ́, Euben 1. . . 359, 360 